\title{
Covid-19 and smart beta
}

\section{A case study on the role of sectors}

\author{
Milot Hasaj ${ }^{1}$ B Bernd Scherer ${ }^{2}$ (1)
}

Accepted: 12 February 2021 / Published online: 15 April 2021

(c) Swiss Society for Financial Market Research 2021

\begin{abstract}
We investigate the role of sectors on the performance of smart beta products during the COVID-19 crisis. Cross-sectional differences in excess returns (versus a market capitalized portfolio) are driven by strong exposures to COVID-19-related industry rotation, rather than to long-term structural causes.
\end{abstract}

Keywords Smart beta $\cdot$ sector performance $\cdot$ Covid-19 $\cdot$ ESG

\section{JEL Classification G12 - G14}

\section{Introduction}

The arrival of COVID-19 created significant stress in equity markets and led to considerable underperformance of smart beta products that often have been sold to investors as cheap alpha as noted in (Amenc 2016). We document smart beta and ESG returns during different stages of the still ongoing crisis and relate them to the underlying (unprecedented) industry rotation induced by the COVID-19 pandemic. The objective of our paper is to find evidence for our conjecture that smart beta returns have been heavily impacted by a crisis specific industry rotation, that cannot be generalized. Rel-

We thank an anonymous referee for valuable contributions

Milot Hasaj: Milot Hasaj is senior quantitative analyst of Lampe Asset Management.

Bernd Scherer: Dr. Bernd Scherer is a Research Associate at EDHEC Risk Institute London and Executive Board Member of Lampe Asset Management.

$凶$ Bernd Scherer

bernd.scherer@edhec.edu

Milot Hasaj

milot.hasaj@lampe-am.de

1 Lampe Asset Management, Düsseldorf, Germany

2 EDHEC Risk Institute, 393-400 Promenade des Anglais, Nice Cedex 3, France 
ative winners (loser) might just have been coincidentally exposed to the right (wrong) sectors. ${ }^{1}$

Where do we derive our conjecture from? Industries differ with respect to the products they produce (business risk) as well as to their capital structure (financial risk, funding risk). We would therefore expect that industries display different sensitivities to macroeconomic factors, i.e., business cycle risks. As every crisis unfolds differently, it leaves its specific marks in the underlying shifts in relative industry performance. (Lamont 2001) and (Hong et al. 2007) show that industry returns can be used to build economic tracking portfolios (i.e., portfolios that track macroeconomic variables like gdp, inflation or consumption). (Hou and Robinson 2006) find that the industry return premium for industry concentration moves systematically with the business cycle and (Chou et al. 2012) show that returns on HML (Fama-French value factor) are driven by inter-industry exposures.

In order to test our conjecture, we follow the work by Paganao et al. (2020) and define incubation, outbreak, fever and (a new) treatment period in Sect. 2. For the two most volatile periods (fever and treatment) we compute ex-post optimal long/short industry portfolios to compute best case industry exposures. We take these weights and backcast them through time to calculate return series for both portfolios. This allows us to explain the variation in excess returns as a function of hidden risk drivers: industry portfolio and market portfolio exposure ${ }^{2}$ in Sect. 3. Given exposures (betas), we employ (Black et al. 1972) two-stage regressions to attribute the performance of smart beta and ESG returns to exposures to market beta and industry rotation in Sect. 4. In Sect. 5 we validate our results using traditional performance attribution. Section 6 concludes.

\section{The year of the virus: from incubation to treatment}

\subsection{Time frames and data}

Our main analysis focuses on the reaction of smart beta portfolios on the performance differential between crisis resilient vs. not resilient sectors during and before the COVID-19 outbreak. We focus on the US market for reasons of data availability, importance in investment portfolios (large weighting of US stocks in global market indexes) as well as academic comparability. In order to be consistent with the latest academic research on the stock market response to the COVID-19 crisis, we follow the taxonomy by Paganao et al. (2020). We study factor returns in the first half of 2020 by closely analyzing three sub-periods: incubation (January 2, 2020, through January 17, 2020), outbreak (January 20, 2020, through February 21, 2020) and fever (February 24, 2020, through March 20, 2020). These periods have been initially identified by Ramelli and Wagner (2020), who document the chronological ordering of events that were crucial in the development of the virus. In contrast to other studies we consider a fourth period (March 23, 2020, through June 30, 2020), that we call the treatment

\footnotetext{
1 We regard our paper as a case study (and reminder) on the importance of industry risk in equity portfolios.

2 See (Auget et al. 2018) on the impact of sector and market risks on smart beta returns.
} 
period. From February 26, 2020, onwards, investors received a constant stream of announcements regarding vaccine developments and treatment options. The Biotech company Gilead Sciences, Inc. announced the initiation of two Phase 3 clinical studies to determine the safety and efficacy of Remdesivir as a potential treatment for COVID19. Weeks later, on March 16, 2020, it was the clinical biotech company Moderna, Inc who announced that the first participant had been dosed in the Phase 1 study of the company's RNA vaccine. In addition, an unprecedented fiscal and monetary stimulus has been provided provided by governments and central banks. ${ }^{3}$

We obtain daily MSCI data for the six smart beta indices: multi-factor, value, momentum, quality, size and minimum volatility (for January 2000 to June 2020). The MSCI factor indices incorporate a long only implementation, use transparent construction methodology, and are (to some extent subject to debate) backed by academic research. They can be directly accessed by investors via exchange traded funds (ETFs). MSCI factor indices are widely used in the investment industry which gives them quasi-benchmark status. Approximately, USD 236 billion in assets are estimated to be benchmarked to MSCI Factor indices. ${ }^{4}$

We also retrieve daily risk free rates from the FRED (Federal Reserve Economic Data - St. Louis FED) database. For the industry representation, we use the S\&P Dow Jones sector indices. S\&P organizes US companies based on their principal business activity in 11 sectors. These sectors represent the Global Industry Classification Standards (GICS) which were introduced by S\&P and MSCI in 1999. The standards seek to offer an efficient investment tool to capture the breadth, depth and evolution of industry sectors. S\&P Indices enjoy great popularity in the investment industry as well. S\&P DJI licenses indices to more than 550 financial institutions worldwide to build investment products linked to their indices. ${ }^{5}$

In the final section of our analysis, where we analyze sector effects on ESG investments, we retrieve four versions of MSCI ESG indices.

1. The ESG Screened Index, which represents a "light" version of ESG integration. It excludes companies of common concern to investors while seeking to maintain a profile similar to market cap indices. ${ }^{6}$

2. The MSCI ESG Enhanced Focus index, which seeks to maximize its ESG profile and reduce its carbon exposure while maintaining risk and return characteristics close to the underlying parent index. ${ }^{7}$

\footnotetext{
3 The Federal Reserve announced its excessive bond buying program on March 23, 2020, to support the economy with a significant impact on equity market performance and sentiment: www.federalreserve.gov/ newsevents/pressreleases/monetary20200323b.htm

4 Source: MSCI: www.msci.com/factor-indexes. Total expense ratio of the investable ETFs related to these indices are not taken into account in the performance of the MSCI indices

5 Source: S\&P Dow Jones Indices (www.spglobal.com).

6 Source: MSCI (www.msci.com/esg-indexes). The screened index is a low tracking error index and aims to exclude companies associated with controversial, civilian, nuclear weapons and tobacco, that derive revenues from thermal coal and oil sands extraction or that are not in compliance with the UNGC principle.

7 The index is constructed through an optimization process, that subject to a low tracking error, aims to maximize its exposure to companies with strong ESG profiles, reduces Carbon Exposure by $30 \%$ compared with the parent index and excludes exposure to Controversial Weapons and Tobacco Producers.
} 
3. The MSCI ESG Leaders Index, which uses a best-in-class approach by only selecting companies that have the highest MSCI ESG Ratings in each sector. Overall the indices target a $50 \%$ sector representation vs. the parent index.

4. The MSCI SRI Index, which currently offers the largest exposure to the highest ESG rated companies by targeting only a $25 \%$ representation versus the parent index. $^{8}$

The data are chosen to resemble the current practice of smart beta and ESG investing in the asset management industry.

\subsection{Industries}

We document the dispersion of industry returns in reaction to the COVID-19 spread in Fig. 1. We start with the fever period. Industries are sorted with respect to their relative performance to build winner and loser portfolios. Loser portfolios consisted of energy stocks (fall in output), financials (low rates and credit exposure), industrials (disruption of supply chains) and real estate (reduced estimates for office space or high end city apartments). Energy stocks had to bear the largest adjustment of $-32 \%$ (rerating of global growth and energy demand). While consumers cut back on discretionary spending (restaurants, vacation, cars,...), expectations on economic growth have been rescaled and the most growth sensitive sectors were repriced. Winners during the fever period have been health care (vaccination and treatment hopes), information technology (business with little client interaction) and consumer staples (stocking up home supplies).

With the advent of monetary policy support and fiscal policy hopes, a new pattern emerged (called K-shaped recovery in the public press). Clear winners were information technology (working from home), discretionary consumption (home improvements including home office infrastructure) and energy (rebound of global growth). Clear losers during the treatment period were defensive, low beta sectors (utilities, consumer staple) as well as financials (rising credit loss provisions).

During fever and outbreak period (absolute) return movements have been elevated and dispersion remained high. This was not the case during incubation and outbreak period. The market moved quickly within the fever period and shifted its focus into the treatment period. It did not anticipate these movements in (the earlier) incubation and outbreak periods.

How does the long-term performance of COVID-19 winner minus loser portfolios look like? To answer this question we build long/short industry portfolios for the fever and treatment period. Portfolios are long the top 5 industries (equal weighted) based on their cumulative performance ranking and short the bottom 5 industries. Once we have the membership lists for our specific portfolios, we can compute their daily returns for the period January 1, 2000, to June 30, 2020. The cumulative performance for ex-post optimal fever $\left(R_{f}\right)$ and treatment $\left(R_{t}\right)$ portfolios are given in Fig. 2. Looking at the data we make two important observations. First, both portfolios offer no long-term reward, i.e., the average return of both portfolio across the last 20 years is close to zero. As

\footnotetext{
8 The best-in-class indices SRI and ESG Leaders both offer higher ESG profiles but also show a higher tracking error and portfolio concentration.
} 


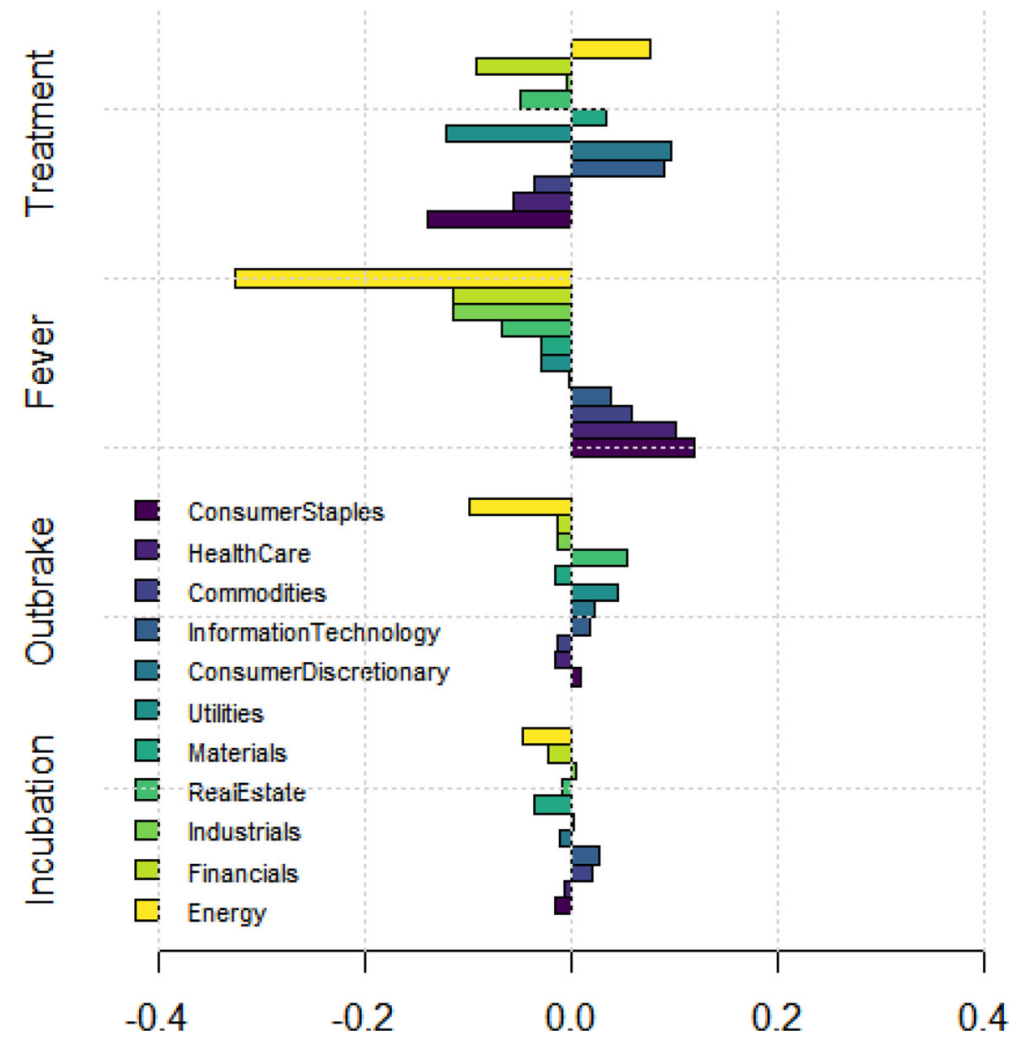

Fig. 1 Industry dispersion. We plot active cumulative industry returns (GICS S\&P classification), i.e., performance relative to market returns for all four non-overlapping periods, from incubation to treatment. Industries are sorted with respect to their relative performance within the fever period

such they are two typical industry long/short portfolios, offering no systematic reward for taking on industry risk. Second, we find the fever period (winner minus loser) portfolio to offer some form of crisis hedge (it performed well in 2008, but lost almost all performance in 2009). However, since the end of the great financial crisis in 2011, this portfolio has performed remarkably well and recovered all its earlier losses. Almost the reverse is true for the treatment portfolio that displays strong performance in the first half of our sample and weak thereafter. The fact that both portfolios performed badly during the 2000 to 2003 tech bubble burst, supports our conjecture, that each crisis unfolds differently and hence creates different sector winner and loser. ${ }^{9}$ Over the whole sample, both portfolios display almost zero correlation (equal to -0.02), while in 2020 (January to June) the correlation dropped to -0.43 . Both portfolios experience annualized volatilities of $12.5 \%$ (fever) and $11.5 \%$ (treatment) for the full sample period (January 1, 2000, to June 30, 2020). The purpose of defining both portfolios is to understand the impact of industry rotation on smart beta and ESG

\footnotetext{
9 "Appendix A" elaborates on this point by showing that indeed industry movements during the COVID-19 crisis have been highly unusual as well as historically unique.
} 


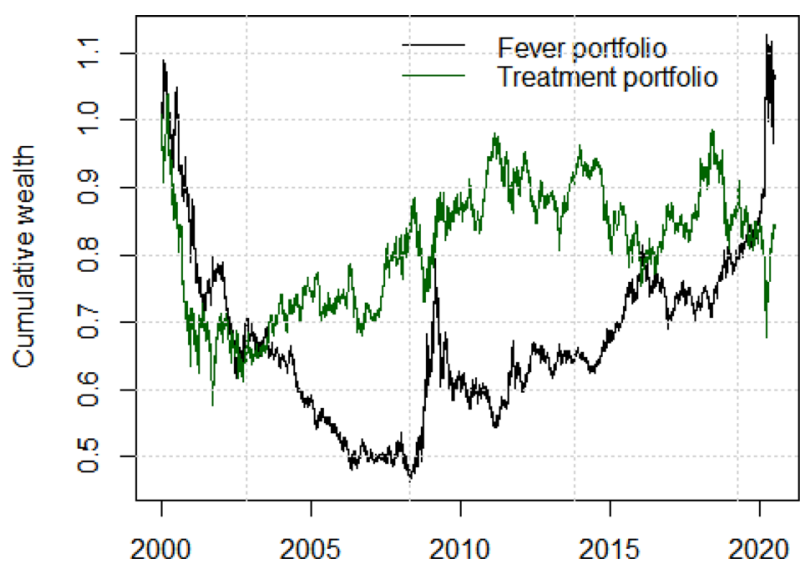

Fig. 2 Industry Rotation Portfolios. We plot the cumulative wealth of two long/short portfolios defined as fever and treatment winner minus loser portfolios. Each portfolio is defined ex-post, i.e., by going long the top (bottom) five industries for the respective time period. Long and short positions each add up to one. All positions are equally weighted

returns. We will use both portfolios in the following sections, to better understand the variation in active smart beta returns.

\subsection{Factors}

How did factors perform during the COVID-19 crisis? We start with smart beta factors. In order not to handpick product providers, we use daily, quasi-benchmark MSCI data (MSCI Multifactor, Value, Size, Quality, Minimum Volatility and Momentum) for the time periods defined above. Figure 3 displays active factor returns (factor return minus market return), where factor returns are sorted with respect to their relative performance within the fever period. We find that quality and minimum volatility investing performed well within the global market sell-off labeled as fever period. This is due to the flight to quality (profitable stocks with limited leverage) and the low beta characteristic of minimum volatility investing. The reverse is true for a period of ample liquidity and rising markets (treatment period), where quality and low volatility are less important stock characteristics. Not surprisingly, small caps and momentum perform well in this scenario. Value stocks did not perform in either fever period (value company typically expose investors to default risk), or treatment period (capital stock of value firms is difficult to adjust to a still pandemic world). We also find in the data what many investors experienced in their portfolios. Diversified multifactor offerings could not outperform in any scenario. Finally, we add ESG data to our analysis, for two reasons. First, asset inflows in products targeting environment (E), social (S) and governance $(\mathrm{G})$ themes are at historical highs, while there is still a big confusion regarding the definition on what ESG represents. Part of this is due to the arrival of preference-based investors, but parts are also due to the ex-post (out)performance results, that seem to attract new investors. Second, the academic literature suggests that ESG represents factor exposures in disguise as argued in (Ang et al. 2020). We 


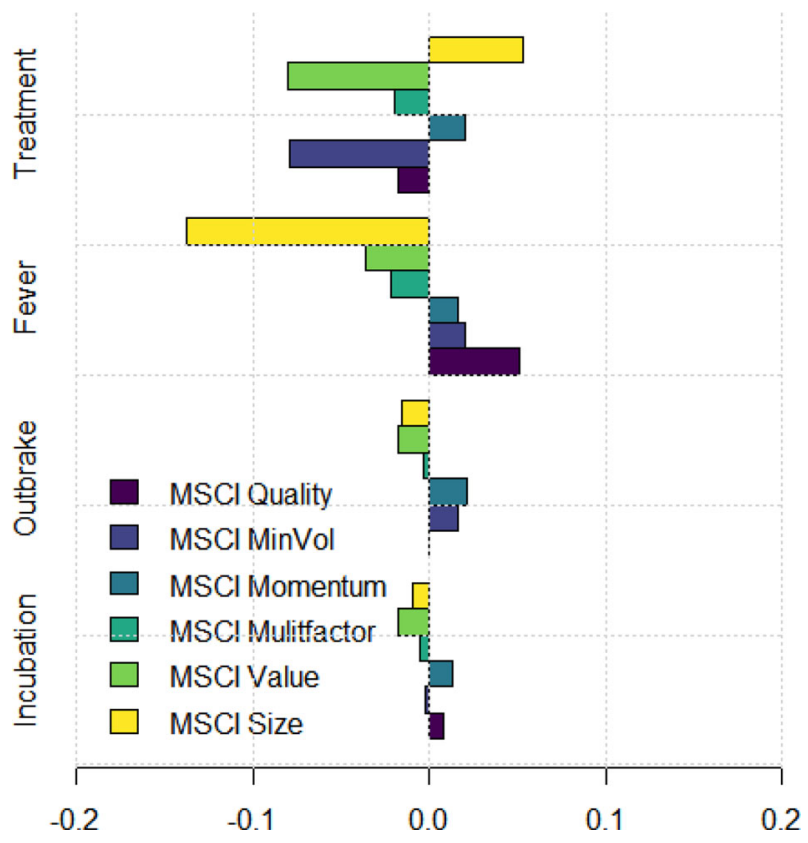

Fig. 3 Factor dispersion. We plot active cumulative factor returns (MSCI factor definitions), relative to market returns for all four non-overlapping periods from incubation to treatment period. Factor returns are sorted with respect to their relative performance within the fever period

plot the active ESG performance (performance relative to market returns) in Fig. 4. In contrast to traditional factors, ESG portfolios outperformed in literally all periods, with the exception of ESG leaders in the treatment period. Performance differences are also a function of risk taking. The ESG SRI index only targets a $25 \%$ market capitalization, which results in large active risks and hence in potential large out- or underperformance. ${ }^{10}$

\section{Industry rotation}

How much of the variance of active returns can be attributed to the industry rotation in 2020? In order to find out, we run a standard OLS-regression in the form of Eq. (1) where we regress factor returns $\left(R_{i}\right)$ against the market return (in excess of the risk free rate, $c$ ) and the long/short period winner portfolios $R_{\text {fever }}$ and $R_{\text {treat }}$.

$$
\left(R_{i}-R_{\mathrm{mkt}}\right)=\alpha+\beta_{m}\left(R_{\mathrm{mkt}}-c\right)+\beta_{\text {fever }} R_{\text {fever }}+\beta_{\text {treat }} R_{\text {treat }}+\epsilon
$$

\footnotetext{
10 The persistent underperformance of the value factor and the simultaneous outperformance of ESG is striking. It is well known that ESG must display risk adjusted underperformance in equilibrium as proven in (Pastor et al. 2020). However, the authors also showed, that an unexpected shift in ESG preferences can lead to transitory ESG outperformance. (Ardia et al. 2020) find confirming evidence. Could this be the reason for values demise?
} 


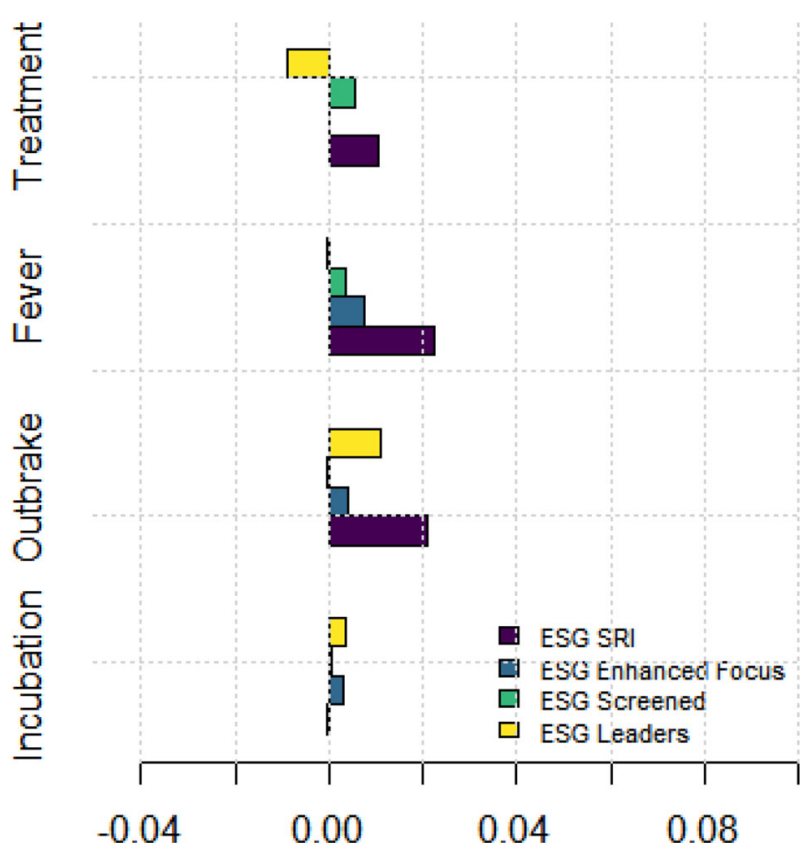

Fig. 4 ESG dispersion. We plot active cumulative ESG returns (MSCI ESG definitions), relative to market returns for all four non-overlapping periods from incubation to treatment period. ESG returns are sorted with respect to their relative performance within the fever period

The dependent variables (left hand variables $i$ ) are daily active returns of factor indices (measured in excess of market returns). We start with Panel (a) in Table 1 for the precrisis period up to December 31, 2019. We find that on average $26 \%$ of their excess return variation is explained by passive exposure to market and industry portfolios. Clearly, there is a substantial portion of hidden (unintended) sector exposure within the set of factor indices. What is the rationale behind these exposures?

We start with the value factor. Despite the fact that MSCI addresses the pitfalls of value investing by using a more diversified definition of value (other than only using book to price), the value factor in the US has been performing poorly since the global financial crisis in 2008. ${ }^{11}$ In our case the value factor's loadings on both industry rotation portfolios are significantly negative. This is not surprising. Value stocks are typically cyclical, high beta, high leverage and low cash companies. Investors have been avoiding those companies not only during the COVID-19 outbreak in 2020 but also prior to the crisis. ${ }^{12}$ The momentum factor shows small and positive exposures on both portfolios. However, only the beta to a backcasted fever portfolio is significant and the explanatory power of our model for momentum is negligible. Momentum

\footnotetext{
11 MSCI uses three descriptors to build the value factor: book value to price, 12-month forward earnings to price and dividend yield. Recent research shows that book-to-price has been an incomplete model of defining the intrinsic company value as argued in (Lev and Srivastava 2019).

12 Ramelli and Wagner (2020) show that especially during the fever period investors saw corporate debt in value firms being more problematic.
} 
follows many themes depending on their prevalence in the data, rather than a time invariant industry exposure. ${ }^{13}$ With its defensive character (targeting companies with low leverage, stable earnings and high profitability) the quality factor displays a highly significant and large (0.186) exposure to the fever portfolio, which essentially is a quality portfolio. ${ }^{14}$ Minimum volatility is by construction a defensive factor. It displays a lack of beta $(-0.14)$ and will perform well when defensive sectors shine. ${ }^{15}$ Finally, we find size to underperform in periods of market stress (beta of -0.24), i.e., when a defensive mix of industries in the fever portfolio does well. The "alpha" for all regressions is either significantly positive or insignificant at worst supporting the longterm value of factor investing.

After having established a baseline for the industry exposure of factors in normal times, we repeat the above regression analysis for the crisis period starting January 1, 2020, through June 30, 2020. Table 1, Panel (b) reports regression results for individual factor indices in 2020. All betas increase considerably and so does the explanatory power of our regression. Between $33 \%$ and $76 \%$ of (active!) return variation is explained by our parsimonious model. Only momentum and quality factor are positively exposed to a fever portfolio. Interestingly the momentum factor has performed well during this crisis, because it has been loading up on quality stocks for quite some time before (given the persistent outperformance of those stocks in the run up to the crisis). The value factor instead has been hit hardest. It did neither perform on the way down (exposure to cyclical sectors), nor on the way up as typical value industries rely on physical capital stocks that are hard to recalibrate to the changing composition of consumer demand (in the treatment period). Minimum volatility displays a negative loading on the treatment portfolio. This does not surprise. However, the equally negative loading on the fever portfolio deserves some explanation. Why did a low volatility portfolio underperform the market, when the fever portfolio was outperforming? Even though the fever portfolio has a defensive character, it comprises not only the defensive (bond proxy) sectors but also high beta - growth sector (stocks) like technology. Hence, the fever portfolio contains the major sectors that the minimum volatility strategy includes, but it goes beyond that having exposure to the sectors that have gone through a regime change in their profitability profile (e.g., glamour stocks) as argued in (Agrawal and Nigro 2020). The size factor loading on a fever portfolio during the crisis is negative, economically large (twice as large as in the pre-crisis period) and statistically significant (beta value of -0.53 with a $t$-value of around 10). Together with a positive loading on the treatment portfolio we interpret this as evidence that excess returns on size are likely to be driven by liquidity. Our results are consistent with academic findings claiming that size is a proxy for a liquidity effect and any size premium can be well captured by a direct liquidity measures as in (Israel et al. 2018). The multifactor portfolio results are self-explanatory, since this

\footnotetext{
13 MSCI uses two descriptors to capture the momentum factor: 6 and 12 months risk adjusted excess return.

14 MSCI uses three descriptors to capture the quality factor: return on equity, debt to equity and earnings variability. There is no commonly accepted definition of quality as argued in (Hsu et al. 2017). Within the MSCI methodology the quality factor selects companies, that sit on the short leg of the value factor. This explains the performance differential between both factors.

15 MSCI constructs a minimum volatility portfolio using an optimization designed to produce an index with the least overall volatility within a given set of sector, country, and factor constraints.
} 
factor simply blends all specific factors. ${ }^{16}$. During the COVID-19 crisis only minimum volatility and quality factor have significantly positive alphas. This is consistent with their crisis hedging properties.

In summary: factors periodically load on (unintended, non-priced) industry risks. This might frustrate investors at times, but should be treated as noise relative to the well documented long-term rewards of factor investing.

\section{The cross section of smart beta returns}

In this section, we want to explain the cross section of smart beta returns. We include ESG returns for economic as well as statistical reasons. Economically, ESG portfolios showed significant outperformance versus US benchmark returns during the COVID19 crisis (see Fig. 4). In addition ESG portfolios are shown by (Ang et al. 2020) to be driven by factor allocations (short small caps and value, long quality and low vol) as well as structural industry positions (long technology, short consumer discretionary and energy). After all ESG portfolios have been the clear winner during the COVID-19 crisis and hence have seen large inflows. Statistically, we increase both the number of cross sectional data points (more degrees of freedom) as well as adding more dispersion across betas. In particular, the latter point is important for reduced estimation error as shown in Ang et al (2020b).

Can different exposures to industry rotation portfolios (fever and treatment) explain the cross section of (out)performance? To analyze this conjecture, we borrow two pass regressions introduced by Black et al. (1972) from the asset pricing literature. First, we regress (first-pass regressions) active asset returns against market excess returns as well as our factor rotation portfolio returns using separate (univariate) regressions. Second, we regress (2nd-pass regression) average outperformance versus betas, to investigate how much of the cross-sectional variation in performance can be attributed to exposures to COVID-19-related industry rotation. We either predict returns, using market betas alone ("CAPM”) in Panel (a)

$$
\hat{r}=\beta_{\mathrm{mkt}} \widehat{\lambda}_{\mathrm{mkt}}
$$

or using market betas as well as sector rotation portfolio betas ("CAPM plus factor rotation) in Panel (b).

$$
\hat{r}=\beta_{\text {mkt }} \widehat{\lambda}_{\text {mkt }}+\beta_{\text {fever }} \widehat{\lambda}_{\text {fever }}+\beta_{\text {treat }} \widehat{\lambda}_{\text {treat }}
$$

Figure 5 graphically summarizes our results. We plot average outperformance $(y$ axis) versus model fitted return ( $x$-axis). Panels differ with respect to beta exposures. The $45^{\circ}$ (blue) line represents the location where predicted model returns and average realized outperformance coincide. If all points would plot on this line, the model would perfectly explain the cross section of returns and the cross-sectional $r$-squared would equate to one. ${ }^{17}$

\footnotetext{
16 We use the MSCI USA Select factor mix as a representation for multifactor investing

17 While two pass regressions have been first applied to large cross-sectional equity data sets, they are now commonly to much smaller numbers of cross-sectional units as shown in (Lettau et al. 2020). Even
} 


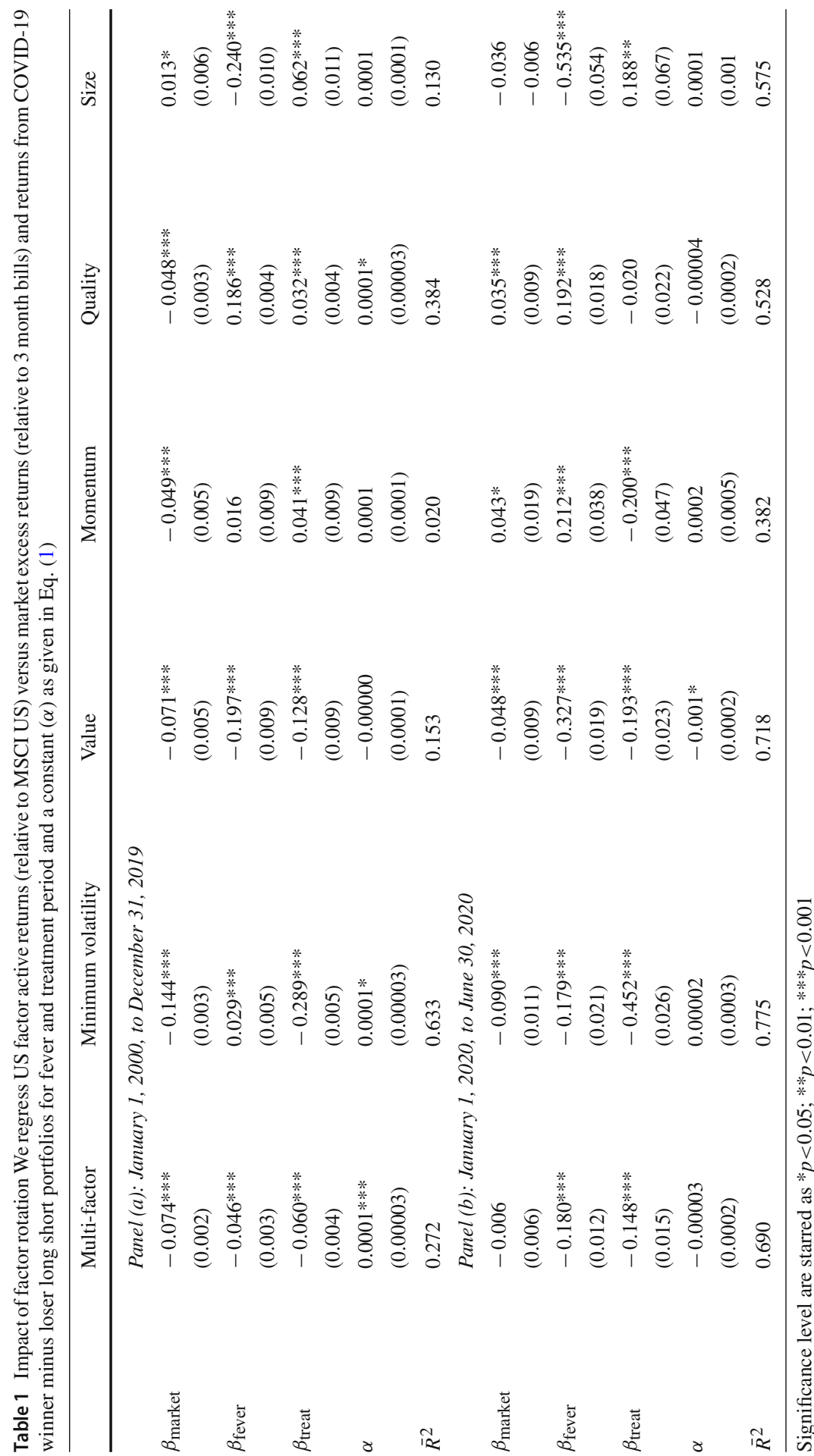


Panel (a): CAPM
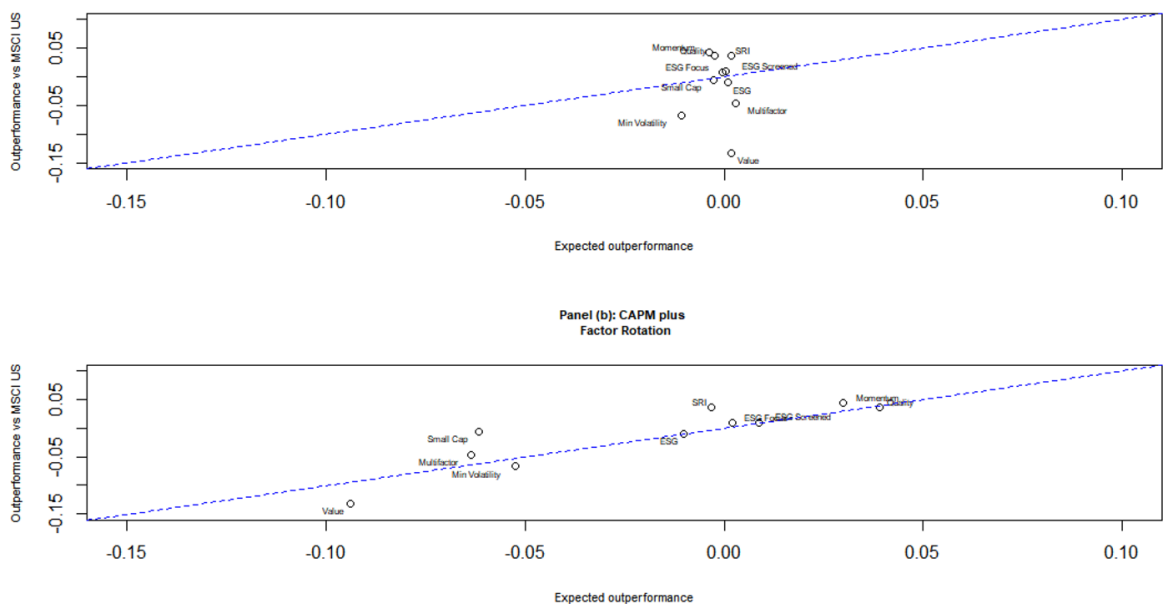

Fig. 5 Cross-sectional return differences: From Fever to Treatment period (February 24 to June 30, 2020). We regress individual betas (from first-stage univariate regressions) against realized average outperformance and plot fitted returns (model expected outperformance) versus realized average outperformance. The $45^{\circ}$ line defines a perfect fit where model expected outperformance equals average outperformance. Return figures are annualized

Using market and industry betas works well for the crisis period in Fig. 5 Panel (b). Average returns are nicely stretched and aligned with model returns, while relying on market beta alone does not explain the dispersion of returns at all in Panel (a). However, we must be careful with eyeballing, as fitted returns also differ with respect to the number of regressors. We need a more formal measure.

It is well known that the adjusted $\bar{R}^{2}$ (adjusted for degrees of freedom, i.e., number of regressors) represents an invalid measure of quality for time series asset pricing model as it measures how much variance of a time series is explained by a set of factors. This is of interest for risk models (it measure the time series variation of returns), but not for modeling the cross section of returns. However, in a cross-sectional model (regress average returns across factor exposures, i.e., betas), the $\bar{R}^{2}$ remains useful. It directly measures how much a model (generating conditional returns) explains the cross-sectional variation in (unconditional) returns. We will hence look at $\bar{R}^{2}$ for above regressions to arrive at a quantitative measure for the improvement of fit (rather than eyeballing). For the heart of the COVID-19 crisis we find $\bar{R}^{2}=-0.11$, when regressing average returns against market betas alone (and no intercept). Excess market betas do not explain the variation in average outperformance at all. However, when adding betas on fever and treatment portfolios we find $\bar{R}^{2}=0.66$. Loadings to fever and treatment portfolios explain the relative performance of smart beta and ESG returns well,. This confirms the much tighter dispersion seen in Panel (b) of Fig. 5, but now

though our sample size is small (10 cross-sectional units) the regression coefficients on fever and treatment portfolio betas are significant at the $1 \%$ respective $5 \%$ level. Adding betas to ESG indices does not change the slope coefficients but materially increases statistical significance. 
adjusted for the increase in the number of regressors (from one to three). ${ }^{18}$ We cannot recover these result for the full sample period. ${ }^{19}$

\section{Validation via performance attribution}

So far our central claim has been that sector movements have dominated the crosssectional return differences across smart beta products during the COVID-19 crisis. The methodology used in the previous sections has many advantages. We did not need to directly observe sector weights within smart beta products. These data are often difficult and expensive to obtain. As we used long/short zero investment portfolios of traded assets we were also able to make use of well-established asset pricing theory. In addition, we did account for different industry rotations across time. Finally, it allowed us to perform risk adjusted performance analysis with respect to betas rather than traditional performance attribution. However, a potential problem for our previous method is spurious correlation between long/short sector portfolios and active returns. If these long/short portfolios proxy for other risk factors, it would make the industry interpretation difficult to maintain.

To tackle this issue, we use traditional (Brinson-type) performance attribution. For this purpose, we gather times series of daily sector weights for the previously analyzed smart beta products as well as for their benchmark (MSCI US). This allows us to approximate the contribution of sector allocation to the benchmark relative performance of smart beta products. We compute returns to sector allocation for a particular smart beta product at time $t\left(r_{\text {allocation, } t}^{\text {smart } \beta}\right)$ as the sum of active industry weights multiplied by market weighted industry returns, $r_{i, t}$

$$
r_{\text {allocation }, t}^{\text {smart }-\beta}=\sum_{i=1}^{11}\left(w_{i, t}^{\text {smart }-\beta}-w_{i, t}^{\mathrm{mkt}}\right) r_{i, t}
$$

Active industry weights are the difference between industry $i$ 's weight in a particular smart beta index $\left(w_{i, t}^{\text {smart }-\beta}\right)$ minus the corresponding weight in the MSCI US benchmark index $\left(w_{i, t}^{\mathrm{mkt}}\right)$. On the 24th of March 2020 the MSCI Value smart beta index was $-21 \%$ underweight the information technology sector (relative to the MSCI USA). At the same time it was about $8 \%$ overweight financial stocks. We can see that returns to sector allocation during COVID-19 can be material.

We provide a more complete picture of differences in active industry weights in Fig. 6. For each smart beta index (color code in legend) and each industry we show boxplots for active industry weights to provide an impression on the cross sectional differences in sector weightings. We use daily data from MSCI for the period February 24 to

\footnotetext{
18 We also test, whether the unbiasedness assumptions (zero intercept) of our regressions are satisfied, but found all regression intercepts statistically irrelevant, when included. In other words our "asset pricing" model consists only of random, but not systematic mispricing.

19 Adding betas for fever and treatment portfolios for the period January 2000 to June 2020 did not improve, but rather lower the models ability to explain unconditional returns. The explanatory power dropped from $26 \%$ to $19 \%$ after adding fever and treatment portfolio betas as explanatory variables for the cross section of returns.
} 


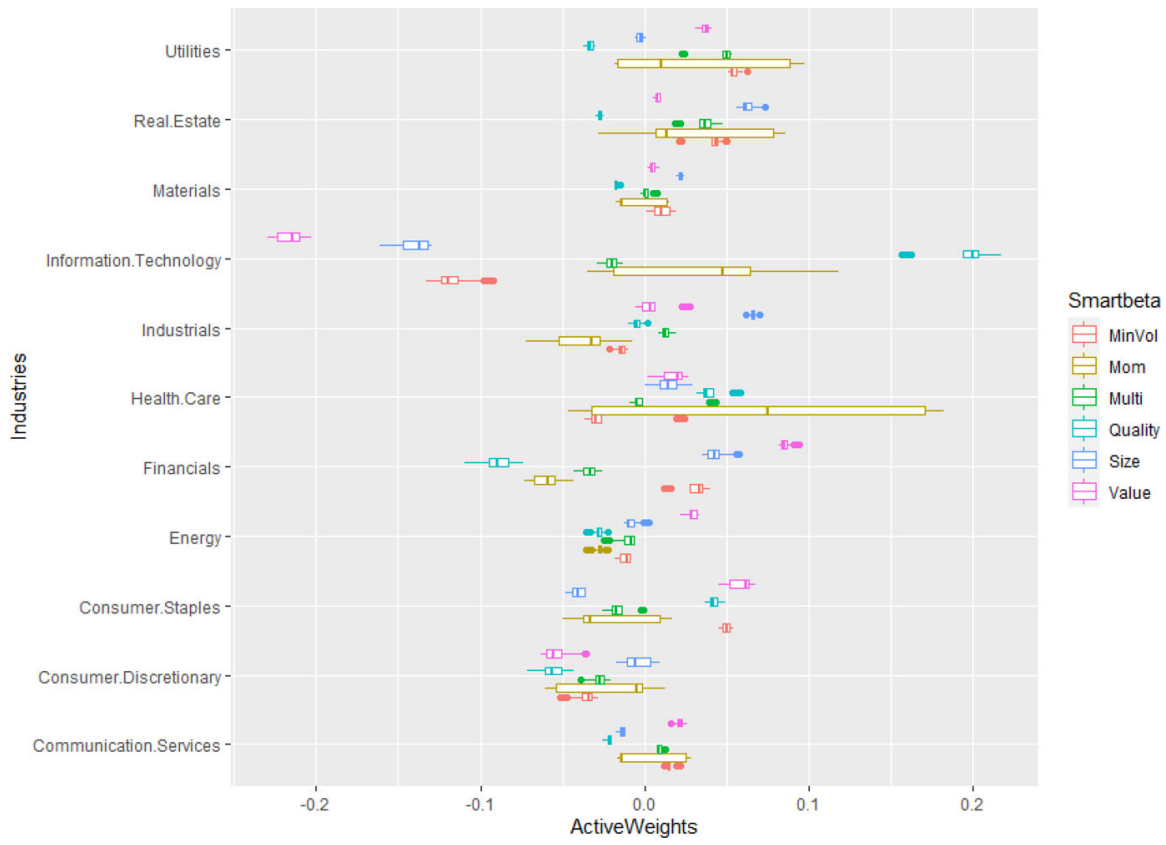

Fig. 6 Distribution of active industry weights For each smart beta index (color code in legend) and each industry we show boxplots for active industry weights to provide an impression of the cross-sectional differences in sector weightings. We use daily data from MSCI for the period February 24 to June 30, 2020.

June 30, 2020. Active industry weights across smart beta concepts are considerably different in every sector, but the most striking difference takes place in information technology, where minimum volatility, size and value display the largest deviations across all sectors and smart beta indices. The same is true for the quality factor. While almost all active weightings are stale, i.e., active weights do not update due to the arrival of new information this is not true for the momentum index. Momentum weights have been updated aggressively due to changes in relative performance as we can see from the width of boxes. This effect has been largest in health care, information technology and utilities.

We already know the active returns for each smart beta product

$$
r_{\text {active }, t}^{\mathrm{smart}-\beta}=r_{t}^{\mathrm{smart}-\beta}-r_{t}^{\mathrm{mkt}}
$$

and therefor define the impact of security selection as the difference between active returns and returns arising from differences in sector allocation. ${ }^{20}$

$$
r_{\text {selection }, t}^{\text {smart } \beta}=r_{\text {active }, t}^{\text {smart }-\beta}-r_{\text {allocation, } t}^{\text {smart }-\beta}
$$

\footnotetext{
20 This is an approximation. In the absence of smart beta specific sector returns, we allocate the entire cross-term (difference in weights times difference in sector performance) to security selection.
} 
Table 2 Performance attribution Performance attribution as derived from Eqs. (4) to (6)

\begin{tabular}{|c|c|c|c|c|c|c|}
\hline & Multi-factor & Minimum volatility & Value & Momentum & Quality & Size \\
\hline & \multicolumn{6}{|c|}{ Panel (a): February 24, 2020, to June 30, 2020} \\
\hline \multirow{4}{*}{$\begin{array}{l}r_{\text {active }}^{\text {smart }-\beta} \\
r_{\text {allocation }}^{\text {smart }-\beta} \\
r_{\text {selection }}^{\text {smart }-\beta} \\
\bar{R}^{2}\end{array}$} & -5.65 & -6.71 & -10.84 & 3.58 & 3.23 & -5.80 \\
\hline & -0.75 & -3.51 & -6.21 & 1.52 & 5.48 & -3.52 \\
\hline & -4.90 & -3.19 & -4.64 & 2.05 & -2.25 & -2.28 \\
\hline & $17.62 \%$ & $63.15 \%$ & $87.51 \%$ & $77.07 \%$ & $61.39 \%$ & $62.68 \%$ \\
\hline \multicolumn{7}{|c|}{ Panel (b): February 24, 2019, to June 30, 2019} \\
\hline$r_{\text {active }}^{\text {smart }-\beta}$ & -3.90 & 1.98 & 2.37 & 2.08 & 1.60 & -1.91 \\
\hline$r_{\text {allocation }}^{\text {smart }-\beta}$ & -0.45 & -0.16 & 1.14 & -0.22 & 1.50 & -0.45 \\
\hline$r_{\text {selection }}^{\text {smart- } \beta}$ & -3.45 & 2.13 & 1.23 & 2.30 & 0.10 & -1.46 \\
\hline $\bar{R}^{2}$ & $10.36 \%$ & 86.87 & $3.99 \%$ & 66.45 & $60.75 \%$ & 27.73 \\
\hline
\end{tabular}

We use daily data for market weighted industry returns, smart beta sector weights and market sector weights for the period from 24th of February to the 30th of June in 2019 and 2020. All data are from MSCI, employing the GICS framework (11 industries)

In addition, regressing $r_{\text {allocation, } t}^{\text {smagt }-\beta}$ against $r_{\text {active }, t}^{\text {smart } \beta}$ provides us with a measure of how much of the variation of a smart betas active returns is due to variations in sector driven return differences.

The results from our analysis are given in Table 2 panel (a) for fever and treatment periods in 2020 . We see that minimum volatility underperforms quality by about $10 \%(3.23 \%+6.71 \%)$, but this difference is mainly due to sector driven performance differences. Sector allocation accounts for around 9\% $(5.48 \%+3.51 \%)$ while security selection (choosing quality stocks versus min vol stocks, instead of choosing quality sectors versus min vol sectors) only adds up to $1 \%(-2.25 \%+3.19 \%)$. These results repeat itself for virtually all smart beta pairs with the exception of momentum. Here, the results are less clear cut as momentum involves more frequent updating, i.e., less static sector exposures. Finally, we see that sector risks explain a large fraction of active return variance for all smart beta indexes apart from multi-factor. The reason for this deviation are the much smaller sector differences of a diversified smart beta product. Smart beta specific sector biases net out after aggregation. We see this from the data in Fig. 6.

How do these results compare with previous periods? For a quick comparison we employ the same time period for 2019 in panel (b). Here, minimum volatility and quality perform almost identical, while sector allocation and security selection show opposite signs. Multi-factor underperforms quality by $5.5 \%$, but only $1.95 \%$ arise from sector allocation. In general, we find that sector allocation returns are considerably smaller in panel (b) than in panel (a), not only in an absolute sense, but also relative to security selection. 


\section{Conclusion}

We find that a substantial fraction of smart beta and ESG returns (as well as their variance) can be attributed to the unprecedented industry rotation that occurred during the COVID-19 fever and treatment period. Covid-19 induced industry rotations explain both the cross section of returns as well as factor variance. Each crisis creates its own industry rotation. Smart beta relies on the identification of systematic risks that investors don't want to hold and therefore those risks demand a risk premium. Investors need to realize that smart beta products expose them to coincidental sector risks that can have dramatic impacts on their performance. Benchmark relative investors that cannot bear the additional volatility arising from mainstream smart beta products, should invest into sector neutral versions of the same smart beta style. Recent (underperformance) is uncomfortable but a poor guide to decide upon the long-run.

\section{Appendix A: Extremeness and similarity}

How unusual have the COVID-19-related events around March (fever period) been relative to previous periods? We employ the framework by Cznois et al. (2020)and compute both a measure of extremeness and of similarity on the sector data. Is this time different? Extremeness (how unusual is a given month's industry return vector given the history of returns as summarized via their historical means and covariance) is defined as

$$
\text { extremeness }\left(r_{t}, \bar{r}\right)=\sqrt{\left(r_{t}-\bar{r}\right)^{T} \Omega^{-1}\left(r_{t}-\bar{r}\right)}
$$

where $r_{t}$ denotes the return in month $t$, while $\bar{r}$ represents the average full sample return (monthly returns from January 2000 to June 2020). The covariance matrix of monthly returns is given by $\Omega$. A month is classified as extreme, if returns in that month are significantly different from average returns in a way that is inconsistent with the covariance matrix. Extremeness is simply the Mahalanobis distance to average returns.

We define similarity (how similar is each individual historical return vector relative to a given month) as

$$
\operatorname{similarity}\left(r_{t}, r_{\mathrm{fever}}\right)=-\sqrt{\left(r_{t}-r_{\mathrm{fever}}\right)^{T} \Omega^{-1}\left(r_{t}-r_{\mathrm{fever}}\right)}
$$

It represents the negative of the Mahalanobis distance between a given month return and the month in question ( $r_{\text {fever }}$, i.e., March 2020). A value of zero describes an identical monthly return profile. Large negative values describe very different return patterns.

The results of our computations are shown in Fig. 7. Panel (a) plots extremeness. March and April, 2020, are highly unusual (i.e., extreme) relative to the history of industry returns. Only September 2008 is equally extreme (in other words: unprecedented). However, we cannot conclude that March 2020 and September 2008 are similar. Both months can be unusual, yet in their own way. We therefore plot our similarity measure in Panel (b). Here, we see that no month comes close to being similar 
Panel (a): Extremeness

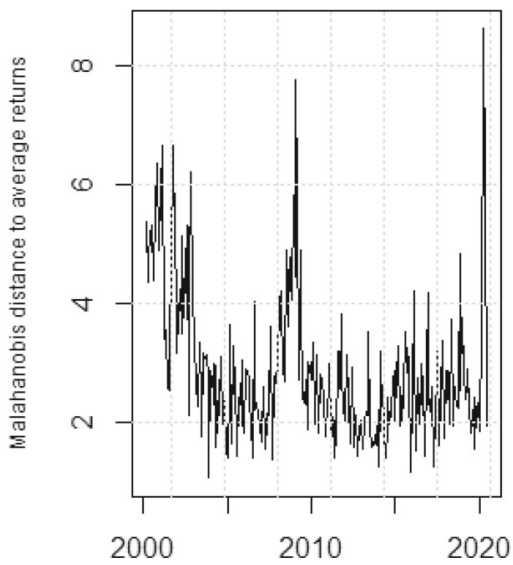

Panel (b): Similarity

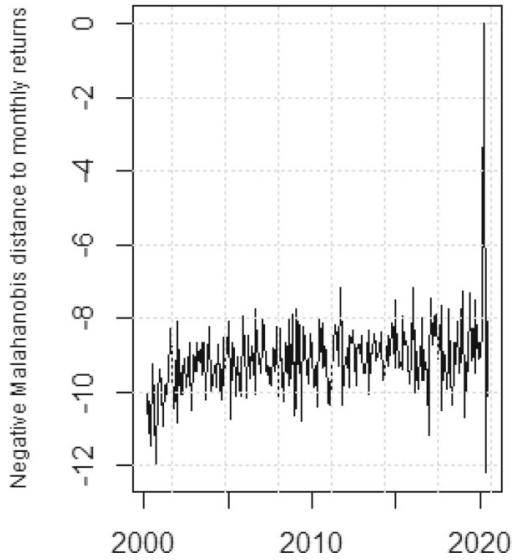

Fig. 7 Extremeness and Similarity We plot measures for extremeness and similarity as defined in Eqs. (7) and (8) for monthly returns between January 2000 and June 2020

to March 2020 (by definition the distance between March 2020 and itself is zero). In other words, the industry rotation seen in March 2020 was both highly unusual as well as unique. The most dissimilar month was April 2020.

Acknowledgements We thank two anonymous referees for their valuable suggestions.

\section{References}

Amenc, N.: J. Bus. Ethics pp. 1-11 (2016)

Ang, A., Chan, Y., Hogan, K., and Schwaiger, K.: ESG in factors, Available at SSRN (2020). https://ssrn. com/abstract $=3522354$

Ang, A., Jun, L., Schwarz, K.: Using stocks or portfolios in test of factor models. J. Financ. Quant. Anal. 55(3), 709-750 (2020)

Ang, A., Hodges, P., Hogan, P., Peterson, J.R.: Factor timing with cross-sectional and time-series predictors. J. Portf. Manag. 44(1), 30-43 (2017)

Agrawal, P., Nigro, M.: The recent decade of drawdown in value: a diagnosis and an enhancement. Two Simga, White Paper (2020)

Ardia D., Bluteau K., Boudt, K., and Ingelbrecht, K.: Climate change concerns and the performance of green versus brown stocks, SSRN (2020)

Auget, D., Amec, N., Goltz, F.: Managing sector risk in factor investing. EDHEC Res. Insights Autumn 2018, 12-19 (2018)

Black, F., Jensen, M.C., Scholes, M.S.: The capital asset pricing model: some empirical tests. In: Jensen, M.C. (ed.) Studies in the Theory of Capital Markets. Praeger, New York (1972)

Chou, P.H., Ho, P.H., Ko, J.C.: Do industries matter in explaining stock returns and asset pricing anomalies. J. Bank. Finance 36(2), 355-370 (2012)

Cznois, M., Kritzman, M., Turkington, D.: Addition by subtraction, a better way to forecast factor returns (and everything else). J. Portf. Manag. 47, 98-107 (2020)

Hong, H., Torous, W., Valkanov, R.: Do industries lead stock markets? J. Financ. Econ. 83, 367-396 (2007)

Hou, K., Robinson, D.T.: Industry concentration and average stock returns. J. Finance 61, 1927-1956 (2006)

Hsu, J., Kalesnik, V., Kose, E.: What is quality? Financ. Anal. J. 75, 44-61 (2017)

Israel, R., Alquist, R., Moskowitz, T.: Fact, fiction, and the size effect. J. Portf. Manag. 45, 34-61 (2018) 
Lamont, O.A.: Economic tracking portfolios. J. Econom. 105, 161-184 (2001)

Lettau, M., Maggiori, M., Weber, M.: Conditional risk premia in currency markets and other asset classes. J. Financ. Econ. 114, 197-225 (2020)

Lev, B., Srivastava, A.: Explaining the recent failure of value investing (2019) https://papers.ssrn.com/sol3/ papers.cfm?abstract_id=3442539

Paganao M., Wagner C. and Zechner, J.: Desaster resilience and asset prices (2020) https://doi.org/10.2139/ ssrn.3603666

Pastor, L., Stambaugh, F., and Taylor, L. A.: Sustainable investing in equilibrium. J. Financ. Econ. (2020)

Ramelli, S., Wagner, A. F.: Feverish stock price reactions to COVID-19. Swiss Finance Institute Research Paper No. 20-12 (2020)

Publisher's Note Springer Nature remains neutral with regard to jurisdictional claims in published maps and institutional affiliations.

Milot Hasaj Milot Hasaj is senior quantitative analyst at a German asset management firm.His work focuses on quantitative research as well on manager selection and portfolio construction for wealth management mandates. He has more than 12 years of experience researching and selecting passive, smart beta and active strategies across all asset classes. Milot holds a masters degree in economics from University of Bonn, Germany.

Bernd Scherer Bernd Scherer is research associate at EDHEC Risk, Nice and executive board member of a German asset management firm. He is an ex professor of finance from EDHEC and worked in senior positions in London, Frankfurt and New York. His academic work has been published in Journals like the Journal of Banking and Finance, Journal of Financial Markets, Journal of Empirical Finance, Review of Economics and Statistics, Journal of Derivatives, etc. 